\title{
Analysis of Antireflection Coatings Using the FD-TD Method with the PML Absorbing Boundary Condition
}

\author{
Junji Yamauchi, Member, IEEE, Makoto Mita, Shin'ichi Aoki, and Hisamatsu Nakano, Fellow, IEEE
}

\begin{abstract}
A step-index optical waveguide with an antireflection coating is analyzed using the finite-difference time-domain (FDTD) method combined with the perfectly matched layer absorbing boundary condition (PML-ABC). It is demonstrated that the numerical simulations having a dynamic range over that for the Mur absorbing boundary condition can be obtained for a singlelayer coating. The analysis of a double-layer coating reveals the transient behavior of reflected fields.
\end{abstract}

\section{INTRODUCTION}

$\mathbf{T}$ THE characteristics of antireflection (AR) coatings have been investigated theoretically or experimentally [1]-[6]. However, experiments are expensive and time consuming, and theoretical solutions are often intractable for complex problems. The numerical modeling has an advantage of applicability to arbitrary structures with sufficient accuracy. Recently, the finite-difference time-domain (FD-TD) method [7], [8] was used to analyze optical waveguides with an AR coating [9], since it is applicable to treating reflection problems. It should be noted that the performance of the absorbing boundary condition $(\mathrm{ABC})$ used with the FD-TD method determines the dynamic range of the numerical simulation. Widening the dynamic range is crucial to the evaluation of low power reflectivities, particularly for a multilayer AR coating.

Several ABC's [10]-[13] have been used for solving unbounded electromagnetic problems with the FD-TD method. Berenger recently published the perfectly matched layer $A B C$ (PML-ABC) [13]. The improved performance of the PML$A B C$ relative to any earlier technique has been verified in optical waveguides such as a three-layer slab one [14] and a one with longitudinal discontinuities [15]. It should be noted, however, that no application has appeared to the evaluation of low power reflectivities from the endface of optical waveguides with AR coatings.

The purpose of this letter is to analyze the reflection problems of optical waveguides with single-layer and double-layer AR coatings using the FD-TD method, and to demonstrate the simulations having an increased dynamic range over that for the Mur-ABC [11]. The calculation shows how the reflected fields are suppressed by the AR coatings as the time is advanced.

Manuscript received August 25, 1995; revised October 24, 1995.

The authors are with the College of Engineering, Hosei University, 3-7-2 Kajino-cho, Koganei, Tokyo 184, Japan.

Publisher Item Identifier S 1041-1135(96)00946-9.

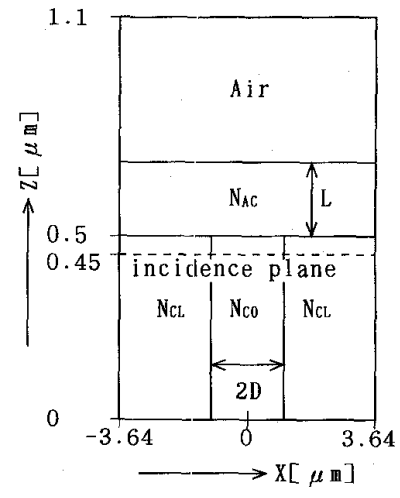

(a)

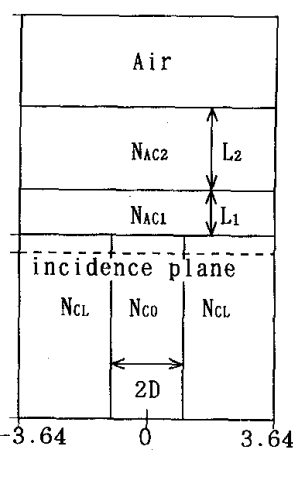

(b)
Fig. 1. Configuration of (a) single-layer AR coating and (b) double-layer AR coating.

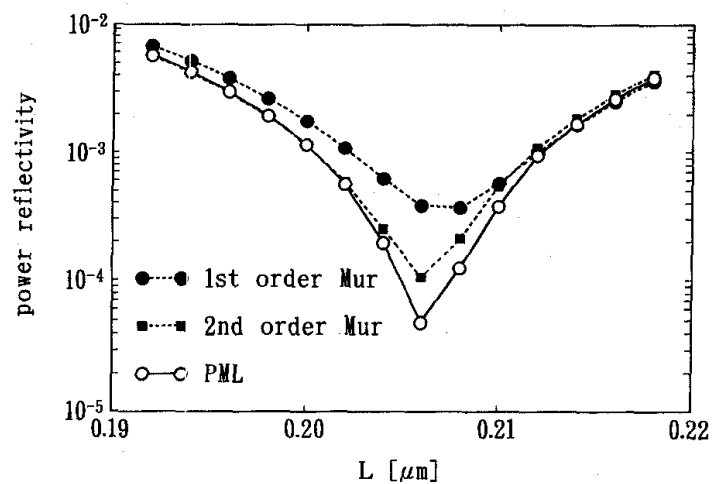

Fig. 2. Comparison in power reflectivity among $A B C$ 's as a function of coating thickness $L$. Single-layer AR coating shown in Fig. 1(a) is treated.

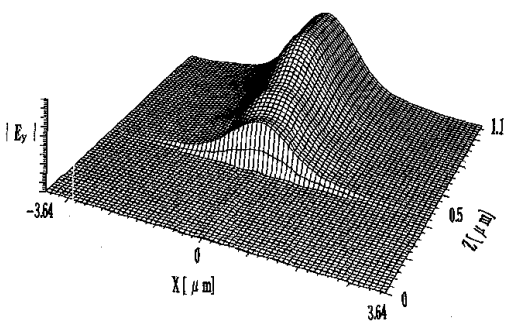

Fig. 3. Steady-state field distribution for double-layer AR coating shown in Fig. 1(b) $(c t=20 \mu \mathrm{m})$

\section{DISCUSSION}

We first analyze the reflection problem for a single-layer AR coating. The configuration to be investigated is a two- 


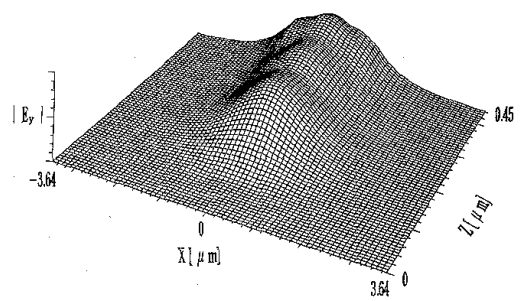

(a)

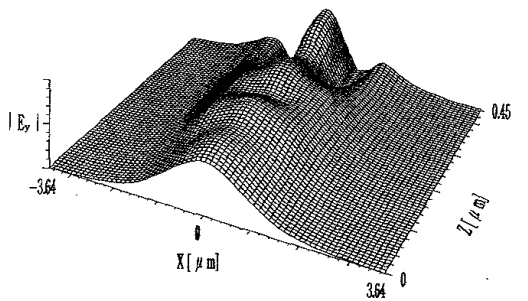

(b)

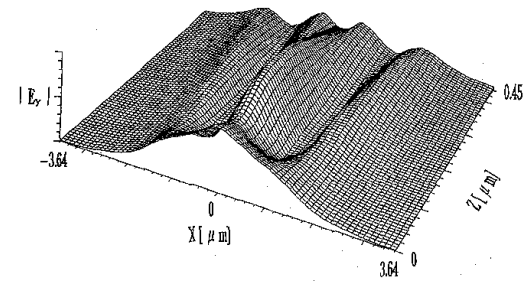

(c)

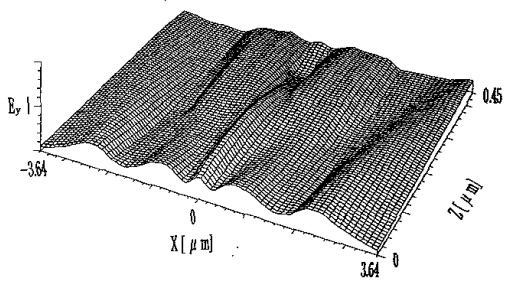

(d)

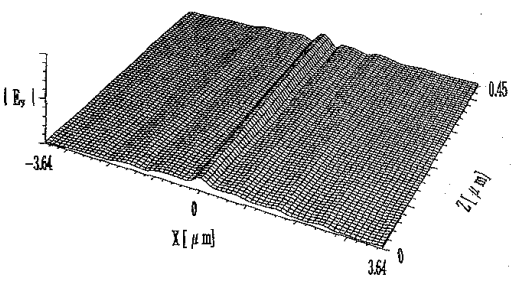

(e)

Fig. 4. Transient behavior of reflected fields in an expanded scale for double-layer AR coating. (a) $c t=1.0 \mu \mathrm{m},(\mathrm{b}) . c t=2.0 \mu \mathrm{m}$, (c) $c t=3.0 \mu \mathrm{m}$, (d) $c t=5.0 \mu \mathrm{m}$ and (e) $c t=20.0 \mu \mathrm{m}$.

dimensional symmetric waveguide whose endface is coated with an AR layer of thickness $L$, as shown in Fig. 1(a). The refractive indices of the core and cladding are chosen to be $N_{C O}=3.6$ and $N_{C L}=3.564$, respectively. A wavelength of $\lambda=1.55 \mu \mathrm{m}$ is used and the normalized frequency is taken to be $V=1.5$ for this analysis. Since the AR mechanism of the coating is considered to be impedance matching between the waveguide and the air, the refractive index of the $\mathrm{AR}$ coating should be $N_{A C}=\sqrt{N_{E}}$, where $N_{E}$ is the effective index of the waveguide. Hence, the refractive index of the AR coating is fixed to be $N_{A C}=1.8938$. The continuous wave simulation of $\mathrm{TE}_{0}$ wave is considered. The $+z$ propagating incident wave is generated on the incidence plane located at $z=0.45 \mu \mathrm{m}$, so that only the reflected field is observed in the region located at $z<0.45 \mu \mathrm{m}$ [8]. The computation parameters are as follows: $\Delta x=D / 10 \approx 0.073 \mu \mathrm{m}, \Delta z=0.002 \mu \mathrm{m}$ and $c \Delta t=0.001 \mu \mathrm{m}$.

The accuracy in the evaluation of power reflectivity depends on the choice of an $A B C$. We, therefore, compare the performance of the PML-ABC with that of Mur-ABC. The PML is a nonphysical lossy medium adjacent to the outer grid boundary (The PML region is located outside the waveguide geometry shown in Fig. 1). Within the PML, the electromagnetic waves rapidly decay without varying the velocity and field impedance, so that the outgoing waves vanish. Throughout this paper, the PML thickness is taken to be 16 cells with a theoretical reflection coefficient $R(0)=$ $10^{-6}$ [13]. The electric conductivities $\sigma(\rho)$ in the PML are defined as a form of $\sigma(\rho)=\sigma_{\max }(\rho / \delta)^{2}$, where $\rho$ is the depth of the PML and $\delta$ is the PML thickness. For reference, the standard first- and second-order Mur-ABC's are tested.

A comparison in the power reflectivity of the fundamental mode among the ABC's as a function of coating thickness $L$ is shown in Fig. 2 for a single-layer coating in Fig. 1(a). From each set of data, it is seen that the power reflectivities become minimums when the coating thickness $L$ is around $0.206 \mu \mathrm{m}$,

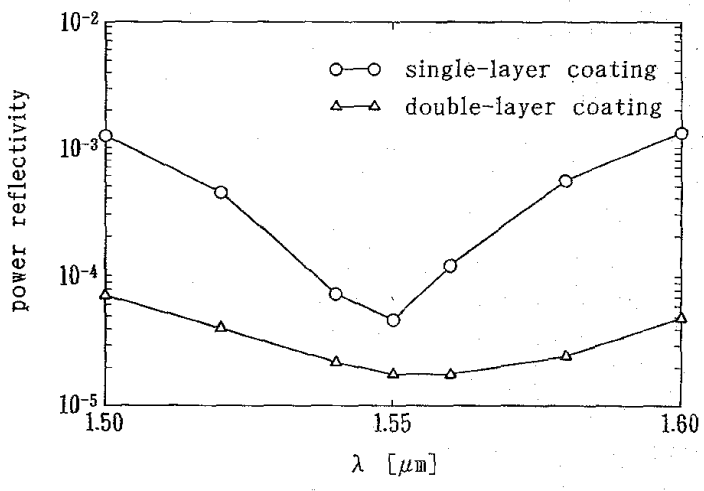

Fig. 5. Power reflectivity as a function of wavelength $\lambda$.

or $\lambda_{g} / 4$. It should be noted, however, that the simulations using the PML-ABC have a dynamic range over that for the Mur-ABC's.

As a further example in which an increased dynamic range is required, we consider the case of a double-layer coating, as shown in Fig. 1(b). The principle of a double-layer coating is the same as that of a single-layer coating. The configuration parameters are as follows: $N_{A C 1}=N_{A C 2} \sqrt{N_{E}} \approx 2.76494$, $N_{A C 2}=1.46, L_{1}=\lambda / 4 N_{A C 1}=0.138 \mu \mathrm{m}$ and $L_{2}=$ $\lambda / 4 N_{A C 2}=0.266 \mu \mathrm{m}$, where $\lambda(=1.55 \mu \mathrm{m})$ is the center wavelength.

Fig. 3 shows the field distribution for a double-layer coating after the transient has passed $(c t=20 \mu \mathrm{m})$. The field is expressed with the sinusoidal steady-state amplitude [16], [17], which can be obtained by the numerical integration of a timedependent signal over one period. It is seen that the reflected fields are successfully suppressed due to the double-layer AR coating. Now, we investigate the propagating field in the reflected field region in more detail. Fig. 4 shows the reflected field distributions in an expanded scale as the time $c t$ is advanced: (a), (b), (c), (d) and (e) are at $c t=1.0 \mu \mathrm{m}, 2.0 \mu \mathrm{m}$, 
$3.0 \mu \mathrm{m}, 5.0 \mu \mathrm{m}$ and $20.0 \mu \mathrm{m}$, respectively. It is observed that the longitudinal discontinuities of refractive index cause reflected fields in Fig. 4(a). As the time is advanced, the field exhibits the complicated transient interference among the reflected fields from the AR coatings, and the reflected fields gradually vanish due to the effects of multiple reflection in the AR coatings. It is shown that the reflected fields become stable in Fig. 4(e).

Fig. 5 shows the power reflectivity as a function of the wavelength $\lambda$. For reference, the result for the single-layer coating ( $L=0.206 \mu \mathrm{m}$ ) is also shown. As expected, the double-layer coating achieves broadband low reflectivities as compared with the single-layer one. It can be said that the FDTD method with the PML-ABC enables us to evaluate low power reflectivities from the endface of optical waveguides with an AR coating.

\section{CONCLUSION}

The characteristics of $\mathrm{AR}$ coatings have been analyzed using the FD-TD method combined with the PML-ABC. For a single-layer coating, the PML-ABC is compared with the Mur-ABC, and the improved performance of the PML-ABC is demonstrated. The transient behavior of the reflected fields and their suppression is revealed for a double-layer coating. The present approach can easily be applied to more complicated structures such as a tilted endface with a multilayer AR coating.

\section{ACKNOWLEDGMENT}

The authors wish to thank Mr. M. Nibe for his assistance in computations.

\section{REFERENCES}

[1] T. Ikegami, "Reflectivity of mode at facet and oscillation mode in double-heterostructure injection lasers," IEEE J. Quantum Electron., vol. QE-8, no. 6, pp. 470-476, 1972.
[2] G. Eisenstein, "Theoretical design of single-layer antireflection coatings on laser facets," Bell Syst. Tech. J., vol. 63, no. 2, pp. 357-364, 1984.

[3] T. Saitoh, T. Mukai, and O. Mikami, "Theoretical analysis and fabrication of antireflection coatings on laser-diode facets," IEEE J. Lightwave Technol., vol. LT-3, no. 2, pp. 288-293, 1985.

[4] C. Vassallo, "Reflectivity of multidielectric coatings deposited on the end facet of a weakly guiding dielectric slab waveguide," J. Opt. Soc. $A m$. A, vol. 5, no. 11, pp. 1918-1928, 1988.

[5] P. C. Kendall, D. A. Roberts, P. N. Robson, M. J. Adams, and M. J. Robertson, "New formula for semiconductor laser facet reflectivity," IEEE Photon. Technol. Lett., vol. 5, no. 2, pp. 148-150, 1993.

[6] D. M. Braun and R. L. Jungerman, "Broadband multilayer antireflection coating for semiconductor laser facets," Opt. Lett., vol. 20, no. 10, pp. 1154-1156, 1995.

[7] K. S. Yee, "Numerical solution of initial boundary value problems involving Maxwell's equations in isotropic media," IEEE Trans. Antennas Propagat., vol. AP-14, no. 3, pp. 302-307, 1966.

[8] S. T. Chu and S. K. Chaudhuri, "A finite-difference time-domain method for the design and analysis of guided-wave optical structures," J. Lightwave Technol., vol. 7, no. 12, pp. 2033-2038, 1989.

[9] J. Yamauchi, T. Ando, and H. Nakano, "Reflection analysis from the endface of optical waveguides using the finite-difference time-domain method," Integrated Photon. Res., pp. 120-123, 1993.

[10] R. Holland, "THREDE: A free-field EMP coupling and scattering code," IEEE Trans. Nucl. Sci., vol. NS-24, no. 6, pp. 2416-2421, 1977.

[11] G. Mur, "Absorbing boundary conditions for the finite-difference approximation of the time-domain electromagnetic-field equations," IEEE Trans. Electromag. Compat., vol. EMC-23, no. 4, pp. 377-382, 1981.

[12] K. K. Mei and J. Fang, "Superabsorption-A method to improve absorbing boundary conditions," IEEE Trans. Antennas Propagat., vol. 40, no. 9, pp. 1001-1010, 1992.

[13] J. P. Berenger, "A perfectly matched layer for the absorption of electromagnetic waves," J. Comput. Phys., vol. 114, pp. 185-200, 1994.

[14] C. E. Reuter, R. M. Joseph, E. T. Thiele, D. S. Katz, and A. Taflove, "Ultrawideband absorbing boundary condition for termination of waveguiding structures in FD-TD simulations," IEEE Microwave Guided Wave Lett., vol. 4, no. 10, pp. 344-346, 1994.

[15] Y. Naka, H. Ikuno, and M. Nishimoto, "Analysis of dielectric slab optical waveguides using the FD-TD method," IEE Japan Tech. Meeting, vol. 95-2, pp. 7-12, 1995.

[16] M. De Pourcq, "Field and power-density calculations in closed microwave systems by three-dimensional finite differences," IEE Proc., Pt. H, vol. 132 , no. 6 , pp. $360-368,1985$.

[17] T. Kashiwa, S. Koike, N. Yoshida, and I. Fukai, "Three-dimensional analysis of patch antenna by Bergeron's method," Trans. IEICE, vol. J71-B, no. 4, pp. 576-584, 1988. 